\title{
BMJ Open Effectiveness and cost-effectiveness of acupuncture with Doin therapy for chronic neck pain: a study protocol for a multicentre, randomised controlled clinical trial
}

\author{
Sook-Hyun Lee, ${ }^{1}$ Jinho Lee, ${ }^{2}$ Yoon Jae Lee, ${ }^{1}$ Me-riong Kim, ${ }^{2}$ Jae Heung Cho, ${ }^{3}$ \\ Koh-Woon Kim, ${ }^{3}$ In-Hyuk Ha ${ }^{1}$
}

To cite: Lee S-H, Lee J, Lee YJ, et al. Effectiveness and cost-effectiveness of acupuncture with Doin therapy for chronic neck pain: a study protocol for a multicentre, randomised controlled clinical trial. BMJ Open 2019;9:e026632. doi:10.1136/ bmjopen-2018-026632

\section{- Prepublication history for} this paper is available online. To view these files please visit the journal online (http://dx.doi org/10.1136/bmjopen-2018026632).

Received 14 September 2018 Revised 29 January 2019 Accepted 6 March 2019

A) Check for updates

(c) Author(s) (or their employer(s)) 2019. Re-use permitted under CC BY-NC. No commercial re-use. See rights and permissions. Published by BMJ.

${ }^{1}$ Jaseng Spine and Joint Research Institute, Jaseng Medical Foundation, Seoul, Republic of Korea

${ }^{2}$ Jaseng Hospital of Korean Medicine, Seoul, Republic of Korea

${ }^{3}$ Department of Korean Rehabilitation Medicine, Kyung Hee University, Seoul, Republic of Korea

Correspondence to

Dr In-Hyuk Ha;

hanihata@gmail.com

\section{ABSTRACT}

Introduction Doin therapy is a manual therapy used in Korean rehabilitation medicine. Recently, the use of acupuncture with Doin has increased in clinics and clinical trials have demonstrated its effects. However, well-designed studies examining the efficacy and costeffectiveness of acupuncture with Doin therapy are rare. Methods and analysis This multicentre, assessorblinded, randomised controlled trial with two parallel groups aims to evaluate the clinical effects and costeffectiveness of acupuncture with Doin therapy. A total of 124 patients (with a neck pain duration of 6 months or longer and a Numeric Rating Scale $\geq 5$ ) will be recruited at five Korean medicine hospitals. Patients will be randomly allocated to acupuncture with Doin therapy $(n=62)$ and acupuncture alone $(n=62)$ for 5 weeks of treatment. This study will be carried out with outcome assessor and statistician blinding. The primary outcome measure will consist of improvement in neck pain using the Visual Analogue Scale at 6 weeks. The secondary outcomes including measures of pain, functional disability, healthrelated quality of life and economic evaluation will be conducted at 6 weeks, and 3, 6, 9 and 12 months after treatment

Ethics and dissemination The project is approved by the Institutional Review Board (IRB) of the Jaseng Hospital of Korean Medicine and the Kyung Hee University Korean Medicine Hospital at Gangdong. Dissemination will occur after the findings from this study are published in other peer reviewed journals.

Trial registration numbers NCT03558178; KCT0003068; Pre-results.

\section{INTRODUCTION}

\section{Background and rationale}

Neck pain is defined as the pain perceived in the dorsal cervical region of the spinal column between the superior nuchal line and an imaginary transverse line through the spinous process of $\mathrm{T} 1 .^{1}$ Pain is categorised as chronic when it lasts longer than 3 months. ${ }^{23}$ Chronic neck pain can be due to

\section{Strengths and limitations of this study}

- Rigorous randomisation and allocation concealment methods will be applied by blinding outcome assessors and statisticians.

- Multicentre trials will provide further evidence on the generalisability of the findings in this study.

- Only acupuncture will be applied to the control group whereas acupuncture with Doin therapy based on the concept of motion style acupuncture treatment will be used in the intervention group.

- Treatment effects on pain, functional disability, health-related quality of life, economic evaluation and long-term cost-effectiveness as well as adverse events will be observed.

various causes such as spinal disc herniation, spondylosis, spinal stenosis, carotid artery dissection and acute coronary syndrome. ${ }^{4}$ In particular, its prevalence rate has been rising due to prolonged poor posture as the overuse of computers and smartphones has become increasingly common. ${ }^{5}$ This also leads to a loss of social productivity due to absence from work or disability in younger individuals and those of economically productive ages. ${ }^{67}$

In traditional Korean medicine, Doin therapy consists of simultaneous breathing and exercise activities. The main goals of Doin therapy are to create a balance between internal and external energies, revitalise the body, mind, and spirit and develop strength and flexibility in muscles and tendons. Although there is no specific definition of exercise therapy, it seems to include the regimen of Doin (導引) and Qigong (氣功).

From the standpoint of modern Korean medicine, Doin therapy is a manual treatment performed by a Korean medicine doctor (KMD). It evaluates the movement 
of the joints or the limited range of motion (ROM) in patients with movement disorders presenting abnormal body movements and disability and then applies passive and active exercises. ${ }^{9} 10$

A systematic review of Doin therapy has reported its effects in various diseases and applications such as improvement of pulmonary function, chronic obstructive pulmonary disease, hepatitis $\mathrm{B}$, postoperative management of coronary artery bypass surgery and improvement of symptoms of irritable bowel syndrome. ${ }^{11}$

In the clinic, Doin therapy is mainly used in combination with acupuncture. The combination of acupuncture with Doin therapy is called motion style acupuncture treatment (MSAT). Recently, MSAT has been increasingly applied in the clinic. Some studies have reported that MSAT is effective in relieving pain in diseases such as low back pain (LBP) and lumbar stenosis. ${ }^{12} 13$

Although studies have investigated the effects of combined acupuncture and exercise therapy in patients with neck pain, no rigorous randomised controlled trials (RCTs) have been performed to confirm the efficacy of Doin therapy in chronic neck pain. ${ }^{14}$ Before conducting this RCT, we performed a pilot study on the efficacy of Doin therapy to improve the quality of the results and provide estimates for sample size calculation. ${ }^{15}$ Based on the results of previous pilot studies on efficacy, we will conduct a rigorous RCT to evaluate the effects of Doin therapy in the treatment of chronic neck pain. This may be the first RCT to evaluate the effectiveness of acupuncture with Doin therapy in the treatment of chronic neck pain. Considering that Doin therapy is used as a combined treatment rather than a single treatment, we divided the treatment groups into Doin with acupuncture and acupuncture alone.

Furthermore, a previous study reported that a comparison of patients with chronic neck pain treated with acupuncture in addition to routine care versus routine care alone resulted a marked clinically relevant benefit and was relatively cost-effective ${ }^{16}$; whereas, there have been no reports on the costs or cost-benefit relationship of acupuncture with Doin therapy compared with acupuncture alone.

The purpose of this study is to clarify the efficacy and cost-effectiveness of acupuncture with Doin therapy or acupuncture alone for chronic neck pain. We will perform a comparative analysis of pain, functional disability, health-related quality of life, economic evaluation and long-term cost-effectiveness.

\section{METHOD AND ANALYSIS}

\section{Study design}

The protocol of this multicentre, assessor-blinded, RCT with two parallel groups was described in accordance with Standard Protocol Items: Recommendations for Interventional Trials 2013 guidelines. ${ }^{17}$ Randomisation will be performed as block randomisation with a random block size of 1:1 allocation. This study is to investigate whether acupuncture with Doin therapy provides more benefits than acupuncture alone. The details of the study design are shown in figures 1 and 2.

\section{Study setting}

This study will be conducted at four community-based hospitals (Jaseng Hospitals of Korean Medicine) and one university-based clinical research centre (Kyung Hee University Korean Medicine Hospital at Gangdong) in Korea. Potential patients will be recruited from hospital websites and using subway advertisements and posters displayed at the clinical trial sites. Additional enrolment and study status will be continually updated.

\section{Eligibility criteria}

All interested patients will first be assessed for eligibility by a trained and registered KMD in accordance with the following inclusion and exclusion criteria in face-to-face consultation.

\section{Inclusion criteria}

1. Neck pain of 6 months or longer.

2. Current Numeric Rating Scale (NRS) for neck pain of 5 or higher.

3. Patients who have agreed to voluntarily participate in the clinical trial and have provided written informed consent.

\section{Exclusion criteria}

1. Patients diagnosed with serious pathology which may cause neck pain (eg, malignant tumour, spinal infection, inflammatory spondylitis).

2. Progressive neurological deficit or severe neurological symptoms (eg, cauda equina syndrome, progressive muscle weakness).

3. Pathologies of non-spinal or soft tissue origin or high severity which may cause neck or radiating arm pain (eg, malignant tumour, spinal infection, inflammatory spondylitis, fibromyalgia, rheumatic arthritis, gout).

4. Other chronic diseases which may interfere with treatment effect or interpretation of results (eg, cardiovascular disorder, renal disease, diabetic neuropathy, dementia, epilepsy).

5. Current treatment with steroids, immunosuppressant medicine, psychiatric medications or other medication which may interfere with treatment results.

6. Patients considered unsuitable or unsafe to receive acupuncture (eg, patients with haemorrhagic diseases, blood clotting disorders, history of anticoagulation medicine intake, severe diabetes with high risk of infection, severe cardiovascular diseases).

7. Patients treated with invasive interventions such as acupuncture or injections, or with medicine that may potentially influence pain such as non-steroidal anti-inflammatory drugs within 1 week prior to the intervention.

8. History of cervical surgery within the past 3 months of the intervention. 


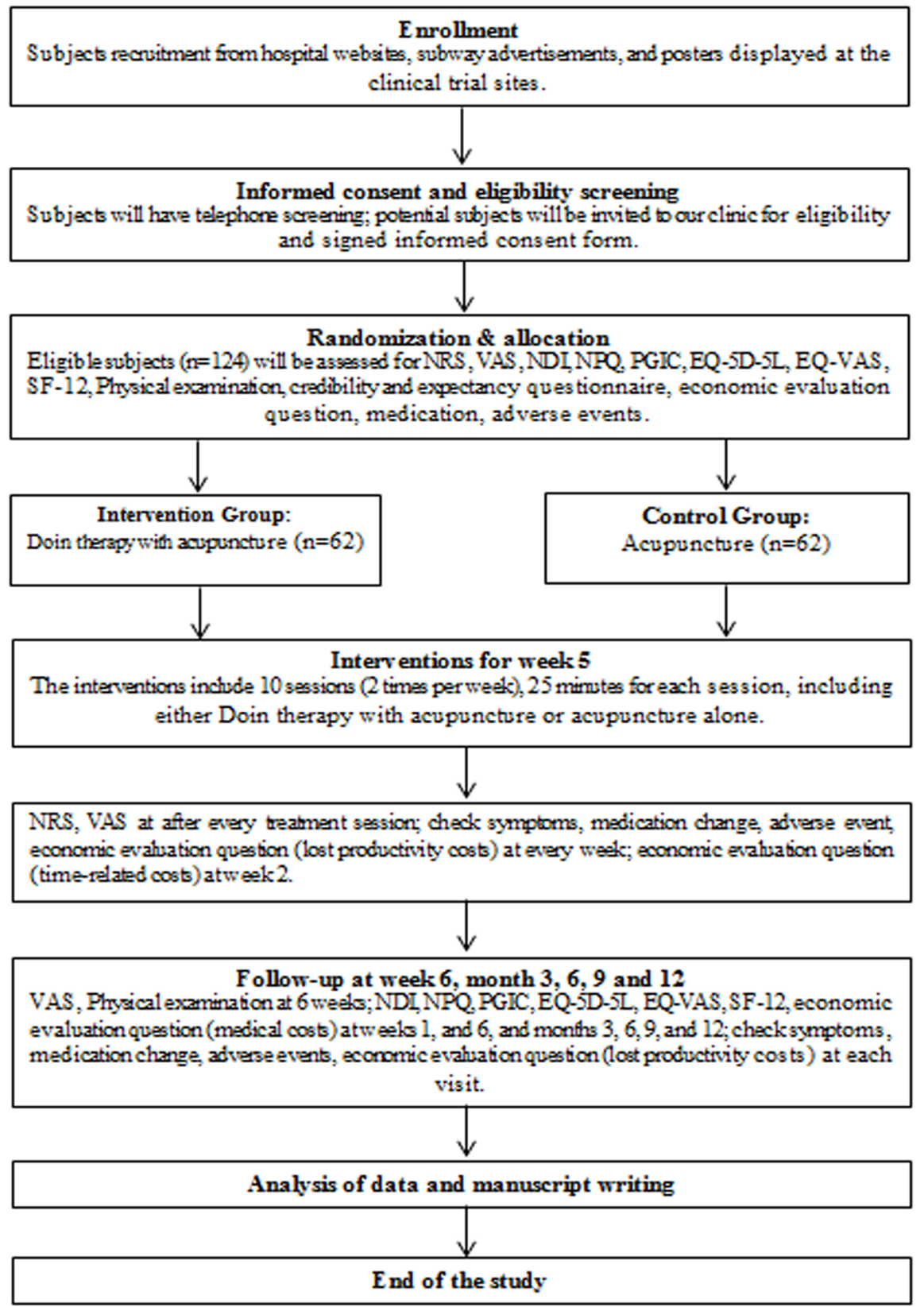

Figure 1 Flowchart of the proposed study. EQ-5D-5L, EuroQol-5 Dimension; EQ-VAS, the EuroQol Visual Analogue Scale; NDI, Neck Disability Index; NPQ, Northwick Park neck pain Questionnaire; NRS, Numeric Rating Scale; PGIC, Patient Global Impression of Change; SF-12, the Short Form Health Survey 12; VAS, Visual Analogue Scale.

9. Pregnancy or plans for pregnancy.

10. Severe psychopathy.

11. Participation in other clinical studies.

12. Inability to give written informed consent.

13. Other reasons rendering trial participation inappropriate as judged by the researchers.

\section{Interventions}

Treatments are provided at one university clinic and four hospitals of Korean Medicine by licensed KMDs with more than 3 years of clinical experience. In order to minimise intervention bias and confusion caused by contextual effects introduced by providers during intervention,
KMDs have been trained to interact with patients with equal enthusiasm for intergroup treatment.

Intervention group: acupuncture with Doin therapy

Sessions of acupuncture with Doin therapy will be conducted in two 15-min visits per week for 5 weeks (a total of 10 sessions). KMD will administer acupuncture at a total of 6-12 acupoints in the upper and middle trapezius areas (mandatory points: both SI15, TE15 and LI16; and selective points: GB20, BL10, GV14, SI14 and EX B2 [Hyeopcheok, Huatuo Jiaji] points at C3-5 levels). Detailed information can be found in table 1 and 2. Acupuncture will be performed with manual stimulation to evoke de-qi 


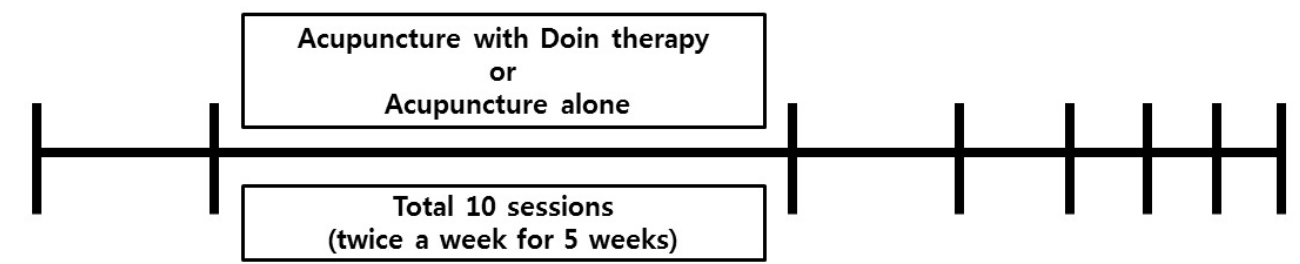

$\begin{array}{ll}\text { Visit 1 } & \text { Visit 2 } \\ \text { - Eligibility } & \text { - Enrollment } \\ \text { - screening } & \text { - Randomized } \\ \text { - Written } & \text { allocation } \\ \text { informed } & \text { - C-spine X-ray } \\ \text { consent } & \text { - Intervention } \\ \text { form } & \text { initiation } \\ & \text { - Initial outcome } \\ & \text { assessment }\end{array}$

Visit 11 Visit 12 Visit 13 16

- Follow-up at - Follow-up at

5 week post week 6 , months

randomization $3,6,9,12$ post

- Primary randomization

endpoint - Outcome

assessment

Figure 2 Study design.

sensation and whether the rotation and movement of the neck is abnormal with the needle inserted will be verified. The side having the smallest movable range on the left or right is referred to as the affected side and the left and right rotational movements proceed based on the movable range of the affected side. It will be repeated 8-10 times in parallel with respiration exercises, the maximum ROM will again be checked and isometric resistance exercises and passive joint movements in the maximum ROM for normalisation of muscles and fascia will be performed. Following the exercise, all needles will be removed and active exercises will be performed for $5-10$ min according to the instructions of the KMD.

\section{Control group: acupuncture alone}

Patients in the acupuncture group will undergo only the acupuncture procedures instead of Doin therapy as in the intervention group. Acupuncture sessions will be conducted for a total of 10 sessions, two times per week for 5 weeks.

\section{Cointerventions}

We will not allow additional treatments (eg, other medication related to pain, Korean medicine, pharmacotherapy, surgery, physical exercise therapy and so on) for the purpose of direct pain relief until the sixth week as the primary endpoint. However, during the study period, acetaminophen (up to $4 \mathrm{~g}$ /day) will be given to all patients as a remedy and drug use will be self-reported. There is no treatment restriction during the follow-up period after 6 weeks.

\section{Outcomes}

Patients in the acupuncture with Doin therapy group and in the acupuncture alone group will be encouraged to complete all treatments within 5 weeks and follow-up will continue for 12 months by the KMD (outcome assessor). The following endpoints will be assessed: (1) Primary endpoint: the Visual Analogue Scale (VAS) of neck pain; (2) secondary endpoints: VAS of radiating arm pain, NRS of neck pain and radiating arm pain, Neck Disability Index (NDI), Northwick Park neck pain Questionnaire
(NPQ), Patient Global Impression of Change (PGIC), physical examination, EuroQol-5 Dimension (EQ-5D-5L), the EuroQol Visual Analogue Scale (EQ-VAS), the Short Form Health Survey 12 (SF-12), economic evaluation (medical costs, time-related costs, lost productivity costs), Credibility and Expectancy questionnaire, drug consumption and adverse events. To ensure consistency in data collection methods, data for all outcomes will be collected via standardised interview by trained blinded assessors. Details of measurement of these outcomes and the time of data collection are provided below.

\section{Primary outcome measurement}

The primary outcome measure is pain intensity. Pain intensity will be measured at baseline as the average neck pain intensity during the previous 3 days using a VAS $(0-100 \mathrm{~mm}, 0=$ no pain and $100=$ worstimaginable pain). ${ }^{18-20}$ We will evaluate the differences between VAS of neck pain at baseline with those after 6 weeks of treatment.

\section{Secondary outcome measurement}

We will evaluate the VAS of radiating arm pain for the previous 3 days at every treatment session and at follow-up at week 6 and months $1,2,3,4,5$ and $6 .{ }^{18-20} \mathrm{In}$ pain measurement using NRS, patients will be asked to rate their neck and upper extremity pain for the past 3 days by selecting a number from 0 to $10(0=$ no pain and $10=$ worst pain possible) immediately after every treatment session and at every follow-up. ${ }^{21}$

The NDI evaluates functional impairment and is a 10-item questionnaire developed to assess the level of disability due to neck pain. ${ }^{22}$ The NDI will be collected by assessors at baseline, and at weeks 1 and 6 , and months 3 , 6,9 and 12 , respectively.

The NPQ evaluates functional impairment for the past 3 days in this study and is a patient-reported outcome of subjective neck pain and pain reduction. ${ }^{23}{ }^{24}$ The NPQ will be measured at baseline, and at weeks 1 and 6 , and months $3,6,9$ and 12 , respectively. 
Table 1 Acupoint locations and standards used for locating acupoints used in this trial ${ }^{\star}$

\begin{tabular}{ll}
\hline Acupoints & Locations of the acupoints \\
\hline SI15 (肩中俞) & $\begin{array}{l}\text { In the upper back region, at the same level } \\
\text { as the inferior border of the spinous process } \\
\text { of the seventh cervical vertebra (C7), } 2 \text { B-cun } \\
\text { lateral to the posterior median line. }\end{array}$ \\
TE15 (天髎) & $\begin{array}{l}\text { In the scapular region, in the depression } \\
\text { superior to the superior angle of the scapula. }\end{array}$
\end{tabular}

\section{Procedures for locating the acupoints}

SI15 is located at the intersection of two imaginary lines: the vertical line of the junction of the lateral one third and the medial two thirds of the line connecting the posterior median line with the medial border of the scapula and the horizontal line inferior to the spinous process of the seventh cervical vertebra (C7).

With the upper limb hanging by the side of the trunk in a seated position, TE15 is located midway between GB21 and SI13.

LI16 (巨骨) $\quad \begin{array}{ll}\text { On the shoulder girdle, in the depression } & \text { In the depression between the two bones lateral to the } \\ \text { between the acromial end of the clavicle and } & \text { suprascapular fossa. }\end{array}$
the spine of the scapula.

GB20 (風池) In the anterior region of the neck, inferior to the occipital bone, in the depression between the origins of sternocleidomastoid and the trapezius muscles.

Note: GB20 is at the same level as GV16.

BL10 (天柱)
same level as the superior border of the
spinous process of the second cervical
vertebra (C2), in the depression lateral to the
trapezius muscle.

GV14 (大椎) In the posterior region of the neck, in the depression inferior to the spinous process of the seventh cervical vertebra (C7), on the posterior median line.

When the head is in a neutral position while the subject is seated, the most prominent site on the posterior aspect of the neck is the spinous process of the C7. Forward flexion of the neck may facilitate palpation of the $\mathrm{C} 7$ spinous process.

Note: Slight rotation of $\mathrm{C} 7$ can be palpated by rotating the head with the neck slightly flexed.

$\begin{array}{ll}\text { SI14 (肩外青) } & \begin{array}{l}\text { In the upper back region, at the same leve } \\ \text { the inferior border of the spinous process } \\ \text { the first thoracic vertebra (T1), } 3 \text { B-cun late } \\ \text { to the posterior median line. }\end{array} \\ \text { EX-B2 (Huatuo Jiaji) } & \begin{array}{l}\text { In the lying about } 0.5 \text { cun bilateral to the } \\ \text { posterior median line, between the third } \\ \text { cervical vertebrae and the fifth cervical } \\ \text { vertebrae. }\end{array}\end{array}$

*Adopted from WHO. WHO standard acupuncture point locations in the Western Pacific Region. WHO standard acupuncture point locations in the Western Pacific Region 2008. B-cun, proportional bone (skeletal) cun.

The PGIC is a method of assessing symptom improvement in 7 levels (1, very much improved; 2, much improved; 3 , slightly improved; 4 , no change; 5 , slightly worse; 6 , much worse; and 7 , very much worse). The PGIC will be measured at week 6 , and months $3,6,9$ and 12 , respectively. ${ }^{25}$ Pain due to movement in cervical ROM will be assessed at weeks 1 and 6 .

The EQ-5D-5L, EQ-VAS and SF-12 are used as tools to evaluate the quality of life of the patients. The tools will be measured at baseline, at weeks 1 and 6 , and months 3, 6, 9 and 12, respectively. The EQ-5D-5L is a tool developed to measure health-related quality of life and is widely used in healthcare ${ }^{26}{ }^{27}$ EQ-VAS is a vertical VAS that takes values between 100 (best imaginable health) and 0 (worst imaginable health), on which patients provide a global assessment of their health. ${ }^{28}$

The SF-12 is a health-related quality-of-life questionnaire consisting of 12 questions that measure eight health domains to assess physical and mental health. Physical health-related domains include general health, physical functioning, role physical and body pain. Mental health-related scales include vitality, social functioning, role emotional and mental health. ${ }^{29} 30$

In economic evaluation, the cost category is largely divided into medical costs, time-related costs and lost productivity costs. ${ }^{31-33}$ Medical costs include direct 


\begin{tabular}{|c|c|c|}
\hline Interventions & Doin therapy with acupuncture & Acupuncture alone \\
\hline Type & $\begin{array}{l}\text { Doin therapy } \\
\text { Medium velocity, frequency } 10-15 \text { times/min } \\
\text { (Velocity and frequency are adjusted according to pain and } \\
\text { limitation of range of motion) } \\
\text { Acupuncture } \\
\text { Manual stimulation to evoke de-qi sensation }\end{array}$ & $\begin{array}{l}\text { Acupuncture } \\
\text { Manual stimulation to evoke de-qi } \\
\text { sensation }\end{array}$ \\
\hline Location & $\begin{array}{l}\text { Doin therapy } \\
\text { The dorsal cervical region of the spinal column } \\
\text { Acupuncture } \\
\text { Total of } 6-12 \text { acupoints in the upper and middle trapezius areas } \\
\text { (mandatory points: SI15, TE15 and LI16; and selective points: } \\
\text { GB20, BL10, GV14, SI14 and EX B2 [Hyeopcheok, Huatuo Jiaji] } \\
\text { points at C3-5 levels). }\end{array}$ & $\begin{array}{l}\text { Acupuncture } \\
\text { Total of 6-12 acupoints in the upper } \\
\text { and middle trapezius areas (mandatory } \\
\text { points: SI15, TE15 and LI16; and } \\
\text { selective points: GB20, BL10, GV14, } \\
\text { SI14 and EX B2 [Hyeopcheok, Huatuo } \\
\text { Jiaji] points at C3-5 levels). }\end{array}$ \\
\hline $\begin{array}{l}\text { Design and } \\
\text { delivery format }\end{array}$ & $\begin{array}{l}\text { Doin therapy } \\
\text { Individualised: cervical region treated with isometric resistance } \\
\text { exercises and passive joint movements with the needle } \\
\text { inserted. Acupuncture needles are removed and active } \\
\text { movement is allowed for } 5-10 \text { min according to the instructions } \\
\text { of medical staff. } \\
\text { Technique position (eg, seated, prone) } \\
\text { Acupuncture } \\
\text { Individualised: acupuncture at } 6-12 \text { points in the upper and } \\
\text { middle trapezius areas. Technique position (eg, seated, prone) }\end{array}$ & $\begin{array}{l}\text { Acupuncture } \\
\text { Individualised: acupuncture at 6-12 } \\
\text { points in the upper and middle trapezius } \\
\text { areas. Technique position (eg, seated, } \\
\text { prone) }\end{array}$ \\
\hline Delivery method & One-on-one treatment visit & One-on-one treatment visit \\
\hline Dose & $10-15 \mathrm{~min}, 2$ visits/week for 5 weeks & $10-15 \mathrm{~min}, 2$ visits/week for 5 weeks \\
\hline
\end{tabular}

expenses incurred by the use of medical facilities and services (official medical cost) and purchase of health foods and medical devices (unofficial medical cost). Non-medical costs are incidental to treatment, and include transportation, patient time and nursing costs. Time-related costs include transportation expenses and time spent visiting the hospital.

Lost productivity cost represents economic loss from decreased work capability because of the disease itself or premature death due to disease. We will use the Work Productivity and Activity Impairment questionnaire to calculate the cost of lost productivity and use it for cost efficiency analysis. ${ }^{34}$ Economic evaluation will be performed to assess cost-effectiveness of the two groups at weeks 1 and 6, and months 3, 6, 9 and 12 .

The Credibility and Expectancy questionnaire will be used to assess treatment expectation on a 9-point Likert Scale ( $1=$ 'not at all'; and 5='somewhat'; to 9='very much') at week 135

For concomitant medication use, the drug type of prescription medicine or rescue medicine (acetaminophen) and adverse events will be recorded at each visit.

\section{Timeline of participants}

Flow and timeline of the proposed study can be found in table 3 .

\section{Patient and public involvement}

Neither the patients nor the public was involved in the development of the research questions, selection of outcome measures, study design or study conduct.

\section{Sample size estimation}

In order to estimate the number of eligible patients, we used the results of unpublished data from a pilot study with the following assumptions: level of significance, $\alpha=0.05$; type II error $(\beta)$ set to 0.2 , with power set to $80 \%$. According to unpublished data from a pilot study using VAS as the main evaluation tool for acupuncture with Doin therapy in neck pain patients, the effect size in comparison to that of controls was 0.55 based on $80 \%$ compliance (predicted rate of completing $>6$ sessions during the 5 -week treatment period); sample size was calculated using G*Power 3.1.7. The effect size resulting from a sample size of 106 participants (53 in each group) was calculated. In this study, analysis of covariance (ANCOVA), which adjusts the baseline pain scores, is the main analysis. Thus, in the preliminary study, the correlation coefficient (CC) between the baseline value and the mean difference value is approximately 0.24 , and adjusting this with $1-\mathrm{CC}^{2}$ and considering $20 \%$ dropout rate the appropriate sample size for this study will be $124 .^{36}$

\section{Assignment of interventions}

Randomisation and allocation concealment

A block randomisation method will be used to achieve balance between the control and treatment groups by stratifying each clinical centre. Participants at each clinical centre will be randomly assigned into either an acupuncture with Doin therapy group or an acupuncture alone group, based on the allocation code.

An independent statistician will generate a table of random sampling numbers using Strategic Applications 


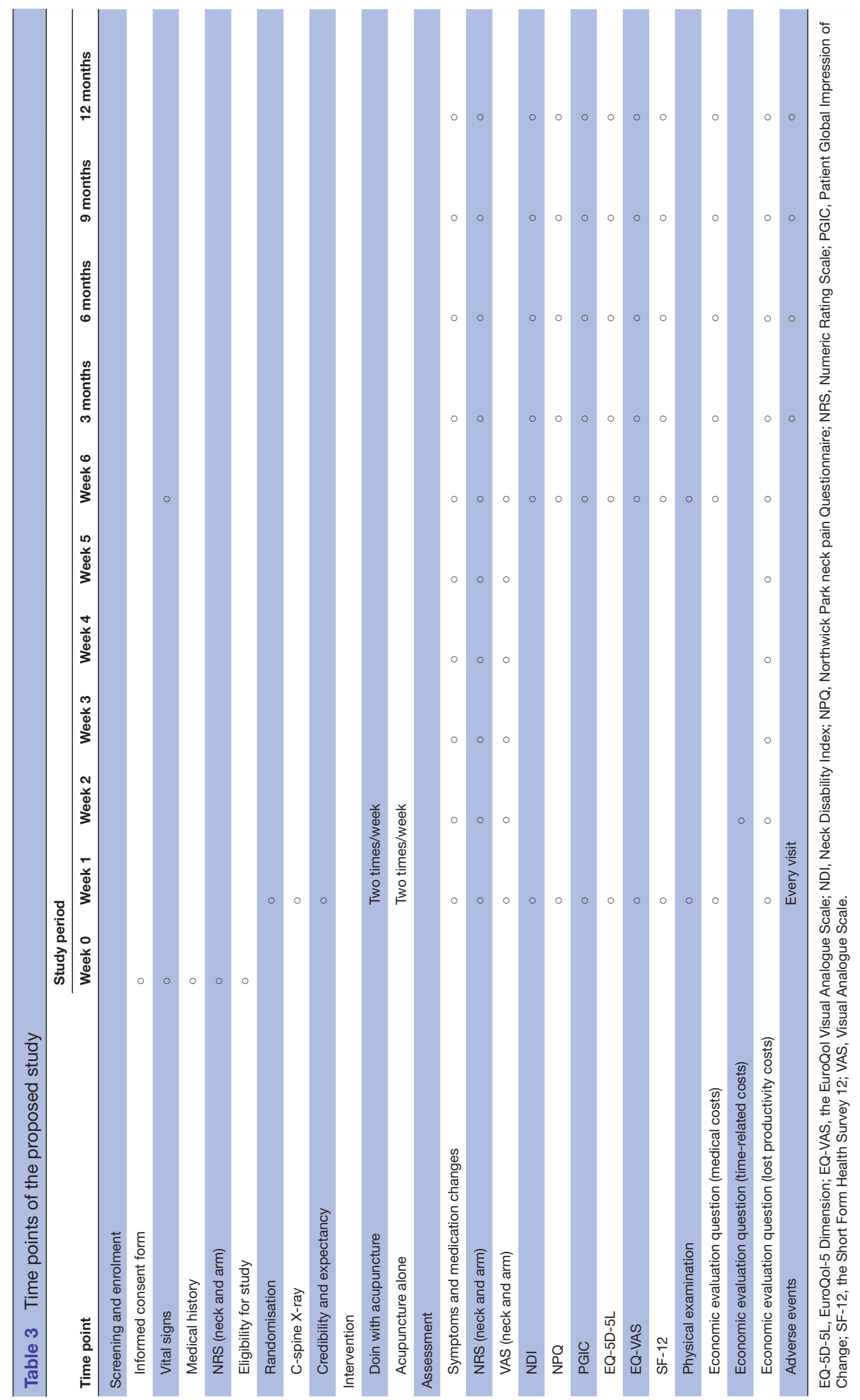

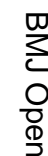

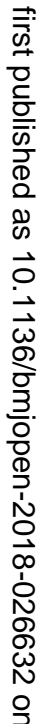

。

产

옹

뭉

咅

@ి

홍

풍

흥.

윰

훙.

옳

웅

을

N

N

远

$\stackrel{0}{\mathbb{D}}$

뭉

市

Q

음 
Software (SAS) V. 9.4 (SAS Institute). The statistician will place the groups to be assigned into a double-layered, opaque envelope in sequence, seal the envelopes and write the numbers in order. The randomisation code will be secured in a double-locked cabinet. As practitioners cannot be blinded by the nature of the intervention, the practitioners will assign the participants to one of the groups by opening the sealed envelopes in order, in front of the participants. The opened envelope will be separately stored in a safe. The practitioner will confirm eligibility before opening the envelope. If the participant does not meet the inclusion criteria, he/she will be removed from the study, and the randomisation will not be performed (ie, the envelope will not be opened).

\section{Blinding}

Blinding of data analysts and outcome assessors will be ensured. The assessors will receive rigorous training in standardised data collection procedures. Data entry personnel external to the research team will be employed to perform data entry such that the data analysts will analyse the data without the need to refer to allocation information.

Given the nature of Doin therapy, with or without acupuncture intervention, blinding of investigators and patients is unsatisfactory. They will not be strongly encouraged from disclosing patients'/own allocation status to data analysts and outcome assessors.

\section{Data management and analysis}

\section{Data management}

We will use an electronic Case Report Form based on the web-based clinical research management systems operated by the Korea Centers for Disease Control and Prevention. We will be establish the standard operating procedures (SOP) before the start of the study and the Case Report Form (CRF), electronic data conversion and SOP guidelines will be provided to the evaluators and researchers of each participating clinical trial institution. The CRF data associated with the outcome indicator will undergo double data entry verification and the researcher will be responsible for the accuracy of data entry for each clinical trial institution. After double-checking that the correct data has been transferred, the data will be blocked to all researchers except statisticians.

\section{Statistical methods and cost-effectiveness analysis}

Intention-to-treat (ITT) and per-protocol (PP) analyses will be conducted to evaluate the final results of this study. Among them, ITT analysis will be used as the main analysis. A multiple imputation method will be used to deal with missing data as the main method, and a last observation carried forward method will be also applied for sensitivity analysis. ${ }^{37}$ The study patients receiving 6 sessions or more for the first 5 weeks will be analysed based on PP analysis.

The sociodemographic characteristics and treatment expectancy of the study patients will be evaluated according to the study groups. The mean (SD) or median (quartile) will be used for the continuous variables whereas the frequencies and percentages will be used for the categorical variables. Student's t-test will be conducted for comparing the differences in the means of the continuous variables, and the $\mathrm{X}^{2}$ test or Fisher's exact test will be also implemented for comparing the differences in the proportions of the categorical variables.

The efficacy evaluation parameters in this study will be the differences in continuous outcomes (ie, NRS, VAS, NDI, NPQ, EQ-5D-5L and SF-12) between baseline and each time point. Baseline values of each outcome and covariant factors showing statistical difference between groups at baseline will be set as covariates and ANCOVA will be conducted with study groups as a fixed factor.

${ }^{38}$ Repeated measures analysis of variance will be used to test the differences in trends at each visit. ${ }^{39}$

At week 6, the areas under the curve (AUC) will be estimated in order to compare the total amount of differences in each outcome between the study groups during the treatment period ( 5 weeks) and the total study period (1 year) and their differences will be compared using Student's t-test.

At each time point, the proportions of the patients whose NRS and VAS values fall below half of baselines will be calculated. The Kaplan-Meier survival analysis will be used to measure the time to the occurrence of this event in the study groups, and the distributions will be compared using the log-rank test.

The Cox model will be used to compare the effects of the treatment on the rate at which pain relief occurs below half of baselines according to various variables (ie, sociodemographic characteristics, physical examination, pain, previous treatment, treatment expectancy and preference, X-ray test and evaluation indices).

The significance that acupuncture with Doin therapy is more effective than acupuncture only will be confirmed through the ANCOVA analysis, which will examine the difference between the VAS of neck pain and the 6-week primary endpoint. On the other hand, we may be concerned with multiple analyses because we will measure various outcomes at different times in secondary outcomes. This is only to explore what parts can have time effects in various categories such as pain, function and quality of life. Significance will be at an $\alpha$-level of $5 \%$, and $\alpha$-level adjustment for multiple testing will not be necessary. All statistical analyses will be conducted using SAS V. 9.4 statistical package (SAS Institute).

For cost-effectiveness analysis, a standard multiplication model will be used to estimate the Quality Adjusted Life Years (QALY), calculated as the area under the linear interpolation of the EQ-5D point trajectory for each individual using the weekly interval. Economic evaluation is performed to compare the cost-effectiveness of acupuncture with Doin therapy with that of acupuncture alone. The primary economic endpoint will be cost per QALY gained. ${ }^{40}$ Primary economic data will be collected for the entire duration of treatment and follow-up. If 
estimates of costs and effects beyond this period are necessary, regression or decision modelling analysis will be employed.

The cost of treatment related to clinical research is calculated by combining the number of treatments and the unit cost. Costs beyond treatment-related study will not be included in the economic evaluation. The unit cost is based on health insurance cost and institutional cost data. QALY will be estimated using the EQ-5D-5L, and the AUC method will be used for QALY calculation. If the time horizon is more than 12 months, the unit of cost will be converted into Korean won (KRW) in 2018 and a discounting rate of $5 \%$ will be applied, based on the Guideline of Health Insurance Review \& Assessment Service in Korea. A social analysis perspective will be used in this study and representative values (eg, averages) of study parameters will be used in baseline analysis. All available distributions and representative values of parameter estimates will be applied in probabilistic sensitivity analysis.

\section{Reporting of adverse effects}

Each institutional clinical researcher will report to the coinvestigator and the subject or caregiver any adverse reactions that may occur after the procedure and provide training for reporting all phenomena that occur after the procedure. Records of the types of symptoms, time, extent, treatment, course and the causal relationship with the procedure, including local, systemic or clinical pathological symptoms after the procedure will be recorded and maintained. In the event of a 'serious adverse event' during the study period, each institutional researcher should describe and evaluate all the symptoms that occurred during the clinical study period when reporting the clinical study. The IRB and the supervising clinical trial centre (Jaseng Medical Foundation) will decide whether to continue or stop the study. Additional safety information should be reported periodically until the adverse event is terminated (such as the disappearance of the adverse reaction or the inability to follow-up). In carrying out the clinical research, the principal investigator of each institute shall carry out all matters in conformity with the Declaration of Helsinki.

\section{Data monitoring and safety monitoring}

To ensure the safety of the subject and the completeness of the research data, the safety of the subject will be examined and the integrity of the data will be reviewed by comparing the case records with the supporting documents. The monitoring plan foresees the conduction of a total of three monitoring visits from the point of selection of the first study patients to the end of the regular monitoring visits and an end of monitoring study visit on the completion of the clinical study. After charting all adverse events reported during the study period, the incidence of adverse events will be determined.
Ethics and dissemination

Patient consent

The KMD, in the role of a screening researcher, will meet face-to-face with the patients. An information sheet will be provided to the patient. The KMD will give the patient time to discuss the possibility of enrolment. For qualified patients who are willing to join, we will obtain formal written consent.

\section{Confidentiality}

All hard copies of study-related information will be securely stored in a restricted access location. Proof that the subject received explanation of the study requirements and provided consent in writing, the date of signing etc, will be recorded on the patient's electronic chart and the contents of the case report will be recorded in writing as well as in the electronic chart of the subject for completeness. All electronic files will be encrypted and password-protected. Only authorised research personnel will be able to access hard copy and electronic data sets.

\section{DISCUSSION}

In this study, acupuncture was selected as a comparator for investigation of the effectiveness and cost-effectiveness of Doin therapy with and without acupuncture in patients with chronic neck pain. Several studies have investigated the cost-effectiveness of acupuncture for chronic neck pain, chronic LBP, headache, osteoarthritis, dysmenorrhoea and allergic rhinitis. In addition, these studies have reported that acupuncture with or without usual care has an acceptable cost-effectiveness. ${ }^{41}$

The application of MSAT has increased in spinal diseases and several papers have reported its effectiveness. A multicentre RCT reported the effects of MSAT in acute LBP patients with severe disability. ${ }^{12}$ Some case studies have reported that motion style treatment was an acute long-term benefit and it was effective on LBP, extruded disc and lumbar stenosis. ${ }^{1342} 43$ To date, there have been no clinical or economic studies comparing MSAT and single acupuncture. Therefore, this study is to evaluate the efficacy and economic efficacy of acupuncture with Doin therapy in chronic neck pain patients compared with patients treated with acupuncture alone.

The strength of this study is that it is the first well-designed, multicentre, assessor-blinded, RCT to evaluate effectiveness and cost-effectiveness of acupuncture with Doin therapy compared with acupuncture alone in patients with chronic neck pain in Korea.

The main shortcoming of this study is that although the evaluator is blinded, we are unable to blind the patient and the therapist due to the nature of the acupuncture treatment. In order to improve the objectivity of the study, evaluation measures such as NRS, NDI, NPQ, EQ-5D-5L, EQ-VAS and SF-12 will be used by blinded evaluators and patients will be given the same form and explanation for 
either treatment arm at the beginning of the study and at follow-up.

Although Korean and Asian patients are typically less resistant to acupuncture treatment and are more exposed to acupuncture treatment, they are not familiar with the combination of active movement with breathing during acupuncture. Therefore, we will be very careful to prevent dropouts of enrolled patients in the acupuncture with Doin therapy group. This is the first study to evaluate the impact of acupuncture with Doin therapy for chronic neck pain to assess its efficacy, safety and economic feasibility. In addition, the data will be used to strengthen Korean health insurance protection in the future.

Contributors Study concept and design: IHH. Drafting of the manuscript: SHL. Critical revision of the manuscript for important intellectual content: JHL, YJL, M-rK, $\mathrm{JHC}$ and K-WK. All authors reviewed the manuscript, approved all the contents and agreed on the submission.

Funding This work was supported by a grant from the Traditional Korea Medicine R\&D Program through the Korea Health Industry Development Institute (KHIDI), funded by the Ministry of Health \& Welfare, Republic of Korea (grant number: HB16C0035). The study design, execution, analysis and interpretation of data, and reporting of results will be conducted separately from the funding source.

Competing interests None declared.

Patient consent for publication Not required.

Ethics approval The study protocol was approved by the Institutional Review Board of Jaseng Hospital of Korean Medicine (JASENG 2018-04-005-001, JASENG 2018-05-006, JASENG 2018-05-007, JASENG 2018-05-008), and Kyung Hee University Korean Medicine Hospital at Gangdong (KHNMCOH 2018-03-003-001).

Provenance and peer review Not commissioned; externally peer reviewed.

Author note 2018.04.10 study protocol (original); 2018.05.14 study protocol (amendment 01); 2018.06.08 study protocol (amendment 02); 2018.06.20 study protocol (amendment 03); 2018.08.20 study protocol (amendment 04). We will periodically update to the trial registration site for important protocol modifications and other changes after the publication of this paper.

Open access This is an open access article distributed in accordance with the Creative Commons Attribution Non Commercial (CC BY-NC 4.0) license, which permits others to distribute, remix, adapt, build upon this work non-commercially, and license their derivative works on different terms, provided the original work is properly cited, appropriate credit is given, any changes made indicated, and the use is non-commercial. See: http://creativecommons.org/licenses/by-nc/4.0/.

\section{REFERENCES}

1. Merskey H, Bogduk N. Classification of chronic pain. Description of Chronic Pain Syndromes and Definition of Pain Terms. 2nd ed. Seattle: ISAP Press, 1994:199-206.

2. Fejer R, Kyvik KO, Hartvigsen J. The prevalence of neck pain in the world population: a systematic critical review of the literature. Eur Spine J 2006;15:834-48.

3. Parisa N, Hooman A, Farnaz K, et al. Acupuncture plus dry needling, an effective treatment method for chronic neck pain. Shiraz $E-M e d J$ 2015;16:e27942.

4. Peter RB, Anita RG, James ME, et al. Clinical Practice Guidelines Linked to the International Classification of Functioning, Disability and Health From the Orthopaedic Section of the American Physical Therapy Association. J Orthop Sports Phys Ther 2017;47:A1-A83.

5. Nandhini T. Cervical neck pain- a review. J. Pharm. Sci. \& Res 2014;6:210-2.

6. Hogg-Johnson S, van der Velde G, Carroll LJ, et al. The burden and determinants of neck pain in the general population: results of the Bone and Joint Decade 2000-2010 Task Force on Neck Pain and Its Associated Disorders.Bone and Joint Decade 2000-2010 Task Force on Neck Pain and Its Associated Disorders. Spine 2008;33:S39-51.

7. Stewart WF, Ricci JA, Chee E, et al. Lost productive time and cost due to common pain conditions in the US workforce. JAMA 2003;290:2443-54.
8. Yang DW, Song TW. A Comparative Study on the Relevance of Doin and Exercise Therapy. Journal of Korean Medicine Rehabilitation 1997;7:533-45.

9. Kim SJ, Kim SJ. Modern Interpretation on Kinesiology of YangsaengDoinbub Presented in [Zhu-Bing-Yuan-Hou-LunYao-Bei-Bing-ZhuHou]. Journal of Korean Medicine Rehabilitation 2014;24:115-30.

10. Choi BS, Ms O. Two cases report of supraspinatus tendon tear treated with the daoyin exercise program combined with Korean medicine treatment. Journal of Korean Oriental Medicine Institute 2016;25:27-36.

11. Hwang $\mathrm{EH}$, Kwon $\mathrm{YK}$, Heo $\mathrm{KH}$, et al. Comparative review of qigong and daoyin as a therapeutic exercise of traditional Korean medicine. Journal of Physiology \& Pathology in Korean Medicine 2013;27:594-601.

12. Shin JS, Ha IH, Lee J, et al. Effects of motion style acupuncture treatment in acute low back pain patients with severe disability: a multicenter, randomized, controlled, comparative effectiveness trial. Pain 2013;154:1030-7.

13. Kim KY, Lee KH, Kim WY, et al. The clinical report on 3 cases of the patient of lumbar stenosis treated with motion style treatment. The Journal of Korea CHUNA Manual Medicine for Spine \& Nerves 2008;3:29-36.

14. Yu Z. Therapeutic effect of exercise acupuncture combined with conventional acupuncture on cervical spondylotic myelopathy. Healthway 2018;17:210.

15. ClinicalTrials.gov. US National Library of Medicine. https:// clinicaltrials.gov/ct2/show/NCT03009071?cond=Neck+Pain\&cntry= KR\&rank=1 (accessed 14 December, 2018).

16. Willich SN, Reinhold T, Selim D, et al. Cost-effectiveness of acupuncture treatment in patients with chronic neck pain. Pain 2006;125:107-13.

17. Chan AW, Tetzlaff JM, Altman DG, et al. SPIRIT 2013 statement: defining standard protocol items for clinical trials. Ann Intern Med 2013;158:200-7.

18. Aitken RC. Measurement of feelings using visual analogue scales. Proc R Soc Med 1969;62:989-93.

19. Gerich J. Visual analogue scales for mode-independent measurement in self-administered questionnaires. Behav Res Methods 2007;39:985-92.

20. Lee JS, Suh KT, Kim Jl, et al. Validation of the korean version of the neck pain and disability scale. Asian Spine J 2013;7:178-83.

21. Farrar JT, Young JP, LaMoreaux L, et al. Clinical importance of changes in chronic pain intensity measured on an 11-point numerical pain rating scale. Pain 2001;94:149-58.

22. Song K-J, Choi B-W, Kim S-J, et al. Cross-Cultural Adaptation and Validation of the Korean Version of the Neck Disability Index. The Journal of the Korean Orthopaedic Association 2009;44:350-9.

23. Yeung PL, Chiu TT, Leung AS. Use of modified Northwick Park Neck Pain Questionnaire in patients with postirradiation neck disability: validation study. Head Neck 2004;26:1031-7.

24. Lee KW, Seo HD, Jung GS, et al. Reliability and Validity of Korean Version Northwick Park Neck Pain Questionnaire in Neck Pain Patients. Korean Professional Physical Therapy Journal 2010;17:68-76.

25. Guy W. ECDEC assessment manual for psychopharmacology (DHEW Publication No. ADM 76-338). Washington DC: U.S. Government Printing Office, 1976.

26. EuroQol Group. EQ-5D-5L User Guide. Rotterdam: EuroQol Group 2011. http://www.euroqol.org/eq-5d/publications/user-guide.html (Accessed 12 Apr 2012).

27. Kim SH, Ahn J, Ock M, et al. The EQ-5D-5L valuation study in Korea. Qual Life Res 2016;25:1845-52.

28. Feng Y, Parkin D, Devlin NJ. Assessing the performance of the EQVAS in the NHS PROMs programme. Qual Life Res 2014;23:977-89.

29. Kim SH, Jo MW, Ahn J, et al. Assessment of psychometric properties of the Korean SF-12 v2 in the general population. BMC Public Health 2014;14:1086.

30. Cheak-Zamora NC, Wyrwich KW, McBride TD. Reliability and validity of the SF-12v2 in the medical expenditure panel survey. Qual Life Res 2009;18:727-35.

31. Gold MR, Siegel JE, Russell LB, et al. Cost-effectiveness in health and medicine: Identifying and valuing outcomes. Oxford University Press 1996:82-134.

32. Berger ML. Health Care Cost, Quality, and Outcomes: ISPOR Book of Terms. Lawrenceville, NJ: ISPOR, 2003.

33. Rascati KL. Essentials of Pharmacoeconomics. Lippincott Williams \& Wilkins, Wolters Kluwer, 2009.

34. Zhang W, Bansback N, Boonen A, et al. Validity of the work productivity and activity impairment questionnaire-general health version in patients with rheumatoid arthritis. Arthritis Res Ther 2010;12:R177. 
35. Devilly GJ, Borkovec TD. Psychometric properties of the credibility/ expectancy questionnaire. J Behav Ther Exp Psychiatry 2000;31:73-86.

36. Borm GF, Fransen J, Lemmens WA. A simple sample size formula for analysis of covariance in randomized clinical trials. J Clin Epidemiol 2007;60:1234-8.

37. Chandan S, Jones MP Bias in the last observation carried forward method under informative dropout. J Stat Plan Inference 2009;139:246-55

38. Feise RJ. Do multiple outcome measures require $p$-value adjustment? BMC Med Res Methodol 2002;2:8.

39. Albert PS. Longitudinal data analysis (repeated measures) in clinical trials. Stat Med 1999;18:1707-32.
40. Torrance GW. Measurement of health state utilities for economic appraisal. J Health Econ 1986;5:1-30.

41. Song YK, Lee HS, Chae YB, et al. A systematic review of costeffectiveness analyses alongside randomised controlled trials of acupuncture. Acupunct Med 2012;30:1-13.

42. Shin JS, Boone WR, Kim PJ, et al. Acute and long term benefits of motion style treatment (MST): Two case reports. International Journal of Clinical Acupuncture 2007;16:85-92.

43. Cho JH, Lee HE, Song JH, et al. The clinical report on 3 cases of the patient of extruded disc treated with motion style treatment. The Journal of Korea CHUNA Manual Medicine for Spine \& Nerves 2007;2:131-40. 